\title{
RECENT DEVELOPMENTS ON THE YAMABE PROBLEM
}

\author{
F. C. Marques \\ Dedicated to Professor Manfredo do Carmo on the occasion of his $80^{\text {th }}$ birthday
}

\begin{abstract}
This article is a brief survey on some recent results about the structure of the set of solutions to the Yamabe Problem. We will discuss a compactness theorem for $n \leq 24$ and some noncompactness results for $n \geq 25$, which together give a complete answer to a conjecture of $\mathrm{R}$. Schoen.
\end{abstract}

\section{Introduction}

Let $\left(M^{n}, g\right)$ be a smooth compact Riemannian manifold of dimension $n \geq 3$. The conformal class of $g$ is the set

$$
[g]=\left\{\tilde{g}=\phi^{2} g: \phi \in C^{\infty}(M), \phi>0\right\}
$$

The Yamabe Problem consists of finding a metric $\tilde{g} \in[g]$ of constant scalar curvature.

This problem is equivalent to showing the existence of a positive solution to the partial differential equation

$$
\Delta_{g} u-c(n) R_{g} u+K u^{\frac{n+2}{n-2}}=0 \quad \text { on } \quad M
$$

where $\Delta_{g}$ is the Laplace-Beltrami operator associated with $g, R_{g}$ is the scalar curvature of $g, c(n)=\frac{n-2}{4(n-1)}$, and $K$ is a constant. If $u>0$ is a solution to the equation (1.1), then the scalar curvature of the metric $\tilde{g}=u^{\frac{4}{n-2}} g$ is constant and equal to $c(n)^{-1} K$.

The Yamabe Problem also has a variational structure. The constant scalar curvature metrics $\tilde{g} \in[g]$ are the critical points of the following energy func- 
tional, also known as the normalized total scalar curvature functional:

$$
Q(\tilde{g})=\frac{\int_{M} R_{\tilde{g}} d v_{\tilde{g}}}{\left(\int_{M} d v_{\tilde{g}}\right)^{\frac{n-2}{n}}} .
$$

It follows from the combined works of Yamabe [34], Trudinger [32], Aubin [2], and Schoen [23], that the Yamabe Problem always admits a minimizing solution.

The conformal classes of compact Riemannian manifolds can be classified in three types, according to the sign of the Yamabe quotient:

$$
Q(M, g)=\inf _{\tilde{g} \in[g]} Q(\tilde{g}) .
$$

If the Yamabe quotient is negative, it follows from the Maximum Principle that the solution (of negative constant scalar curvature) is unique. If the Yamabe quotient is zero, the solution (of zero scalar curvature) is unique up to a constant factor.

The structure of the set of solutions in the positive Yamabe quotient case can be very rich though. The simplest and most important example is given by the standard sphere $\left(S^{n}, g_{0}\right)$. It is special because the standard sphere is the only compact manifold which admits a noncompact group of conformal transformations $\operatorname{Conf}\left(S^{n}\right)$. It follows from a theorem of Obata (see [21]) that the full set of solutions to the Yamabe Problem in this case is given by

$$
\left\{\lambda \psi^{*}\left(g_{0}\right): \lambda \in \mathbb{R}_{+}, \psi \in \operatorname{Conf}\left(S^{n}\right)\right\} .
$$

Every solution is minimizing in this particular example.

In [25], R. Schoen studied the example of $S^{1}(L) \times S^{n-1}$ with the product metric ( $L$ denotes the length of the circle factor), and verified the existence of a large number of high energy solutions with high Morse index. In fact, a theorem of Pollack ([22]) shows that every compact Riemannian manifold of positive scalar curvature can be perturbed, in the $C^{0}$ topology, to have as many solutions as desired. These solutions generally have high energy and index. 
In a topics course at Stanford in 1988 (see also [26] and [27]), motivated by the study of the locally conformally flat case, R. Schoen proposed the following conjecture:

\section{Compactness Conjecture}

The set of solutions to the Yamabe Problem, in the positive Yamabe quotient case, is always compact unless the manifold is conformally equivalent to the standard sphere.

The cases which were covered in the Stanford notes are the locally conformally flat case, published in [26] and the three dimensional case, the argument for which is in the paper of Schoen and Zhang [31] (used there to establish a single simple point of blow-up for the prescribed scalar curvature problem on $S^{3}$ ). In dimensions 4 and 5 , the conjecture was proved by O. Druet (see [8]).

It follows from basic arguments in blow-up analysis that non-converging sequences of solutions to the Yamabe Problem have to concentrate and form bubbles at some points of the manifold, referred to as blow-up points. The main difficulty in establishing compactness in dimensions greater than or equal to 6 consisted in proving a related statement, known as the Weyl Vanishing Conjecture, concerning the location of possible blow-up points:

If $\bar{x} \in M$ is a blow-up point of a sequence of solutions $\tilde{g}_{\nu}=u_{\nu}^{\frac{4}{n-2}} g$ to the Yamabe Problem, then the Weyl tensor of the metric g should satisfy

$$
\nabla^{k} W_{g}(\bar{x})=0
$$

for all $0 \leq k \leq\left[\frac{n-6}{2}\right]$.

Over the past several years many people have worked on these problems. It follows from the works of the author ([18]) and Y. Y. Li and L. Zhang ([14]) that compactness holds for $n \leq 7$ in general, and for arbitrary $n$ under the assumption that the Weyl tensor vanishes nowhere to second order. In [15], Li and Zhang proved compactness for $n \leq 11$. 
We should also point out that non-smooth blow-up examples were obtained by A. Ambrosetti and A. Malchiodi in [1], and by M. Berti and Malchiodi in [3]. In [9] O. Druet and E. Hebey have also obtained blow-up examples for Yamabe-type equations.

In a surprising paper ([6]) Simon Brendle has constructed examples of $C^{\infty}$ metrics on spheres of dimension at least 52 for which the compactness statement fails. In a subsequent paper ([7]) Brendle and the author were able to extend these examples to the dimensions $25 \leq n \leq 51$. Finally, in [11], M. Khuri, R. Schoen and the author have established compactness if $n \leq 24$.

The main goal of this survey article is to give an overview of the results and arguments of the papers [11], [6], and [7]. Their results put together give the following answer to the Compactness Conjecture:

The Compactness Conjecture is true if and only if $n \leq 24$.

We should point out that another recent important development in the Yamabe Problem is the proof of convergence of the Yamabe flow in high dimensions by S. Brendle (see [5]). See also [4] for a related survey on these developments.

\section{Compactness theorem}

Let $\left(M^{n}, g\right)$ be a smooth compact Riemannian manifold of dimension $n \geq 3$ and positive Yamabe quotient.

For any $p \in\left[1, \frac{n+2}{n-2}\right]$ we define

$$
\Phi_{p}=\left\{u>0 \mid L_{g} u+K u^{p}=0 \text { on } M\right\},
$$

where $L_{g}=\Delta_{g}-c(n) R_{g}$ is the conformal Laplacian operator. Although the geometric problem corresponds to the exponent $p=\frac{n+2}{n-2}$, critical with respect to the Sobolev embeddings, the consideration of the subcritical solutions will 
be useful for the purposes of computing the total Leray-Schauder degree of the problem.

The main theorem of [11] is:

Theorem 2.1. Let $\left(M^{n}, g\right)$ be a smooth compact Riemannian manifold of dimension $3 \leq n \leq 24$. If $\left(M^{n}, g\right)$ has positive Yamabe quotient and is not conformally diffeomorphic to $\left(S^{n}, g_{0}\right)$, then for any $\varepsilon>0$ there exists a constant $C>0$ depending only on $g$ and $\varepsilon$ such that

$$
C^{-1} \leq u \leq C \quad \text { and } \quad\|u\|_{C^{2, \alpha}} \leq C,
$$

for all $u \in \cup_{1+\varepsilon \leq p \leq \frac{n+2}{n-2}} \Phi_{p}$, where $0<\alpha<1$.

The proof of this theorem is by contradiction and follows the strategy outlined in the notes of R. Schoen ([24]). The basic idea is to use the Pohozaev Identity as an obstruction tool in order to rule out the formation of bubbles at blow-up points.

Proposition 2.2 (Pohozaev Identity, [30]). Let $\left(\Omega^{n}, g\right)$ be a Riemannian domain, $n \geq 3$. If $X$ is a vector field on $\Omega$, then

$$
\frac{n-2}{2 n} \int_{\Omega} X\left(R_{g}\right) d v_{g}+\int_{\Omega}\left\langle\mathcal{D}_{g} X, T_{g}\right\rangle d v_{g}=\int_{\partial \Omega} T_{g}\left(X, \eta_{g}\right) d \sigma_{g} .
$$

Here $T_{g}=$ Ric $c_{g}-\frac{R_{g}}{n} g$ is the traceless Ricci tensor, $\left(\mathcal{D}_{g} X\right)_{i j}=X_{i ; j}+X_{j ; i}-$ $\frac{2}{n} d i v_{g} X g_{i j}$ is the conformal Killing operator, and $\eta_{g}$ is the outward unit normal to $\partial \Omega$.

The strategy has both a local and a global aspect. The global aspect involves the so-called Positive Mass Theorem of General Relativity. This theorem has been established by Schoen and Yau [28] for general manifolds in dimensions $n \leq 7$. In [33] E. Witten established it in any dimension for spin manifolds, while the locally conformally flat case was handled by a special argument in [29]. The general higher dimensional version has been recently announced by Lohkamp [17]. 
In order to illustrate the idea let us for simplicity restrict ourselves to the critical exponent $p=\frac{n+2}{n-2}$. Suppose there exists a sequence $u_{\nu} \in \Phi_{\frac{n+2}{n-2}}$ such that $\max _{M} u_{\nu}=u_{\nu}\left(x_{\nu}\right) \rightarrow \infty$ as $\nu \rightarrow \infty$. Suppose $\bar{x}=\lim x_{\nu}$. Since the scalar curvature of $g_{\nu}=u_{\nu}^{\frac{4}{n-2}} g$ is constant, the Pohozaev identity applied to the geodesic ball $B_{\delta}\left(x_{\nu}\right)=\left\{p \in M: r=d_{g}\left(x_{\nu}, p\right) \leq \delta\right\}$, endowed with the Riemannian metric $g_{\nu}$ and the radial vector field $X=r \frac{\partial}{\partial r}, r=d_{g}\left(x_{\nu}, \cdot\right)$, yields

$$
\int_{B_{\delta}}\left\langle\mathcal{D}_{g_{\nu}} X, T_{g_{\nu}}\right\rangle d v_{g_{\nu}}=\int_{\partial B_{\delta}} T_{g_{\nu}}\left(X, \eta_{g_{\nu}}\right) d \sigma_{g_{\nu}} .
$$

It turns out that the boundary integrals in the identity (2.1), when apropriately normalized, converge to a quantity which can be bounded above by $-m$, where $m$ is the mass of the asymptotically flat and scalar flat metric $\hat{g}=G_{L}^{\frac{4}{n-2}} g$. Here $G_{L}$ denotes the Green's function of the conformal Laplacian with pole at $\bar{x}$. The vanishing of the Weyl tensor to order $\left[\frac{n-6}{2}\right]$ at $\bar{x}$ is necessary in order for the mass of $\hat{g}$ to be well-defined.

Since $\left(M^{n}, g\right)$ is not conformally equivalent to the standard sphere, the metric $\hat{g}$ is not flat, and hence $m>0$ by the Positive Mass Theorem. The contradiction will come if we can establish the nonnegativity of the corresponding limit of the interior integrals of (2.1). The study of the sign of the interior integral constitutes the local aspect of the strategy, and it turns out that when it works the Weyl vanishing at the blow-up point also follows.

The first step is to obtain sharp approximations of the blowing-up sequence of solutions in a neighborhood of the blow-up point. This is achieved by establishing optimal pointwise estimates which generalize the ones obtained by the author in [18]. These estimates assume the blow-up point is isolated simple. After the strategy is carried out with success for that particular case, the results can be used to handle the more general case of multiple blow-up by scaling arguments.

The important point of the estimates of [11] is that in high dimensions it is necessary to perform a refined blow-up analysis and go beyond the rotationally symmetric first approximation (standard bubble). The approximate solutions 
used are the same ones introduced by S. Brendle in [5] to generalize the results of Aubin ([2]) and of Hebey and Vaugon ([10]).

If $\left(x_{1}, \ldots, x_{n}\right)$ are normal coordinates centered at $x_{\nu}$, we can write the components of the metric $g$ in the form

$$
g_{i j}(x)=\exp \left(h_{i j}(x)\right)
$$

and look at the Taylor expansion of $h_{i j}$ around the origin:

$$
h_{i j}(x)=H_{i j}(x)+O\left(|x|^{d+1}\right),
$$

where $d=\left[\frac{n-2}{2}\right]$. It is convenient to work in conformal normal coordinates (see [13]) to simplify the computations. In that case $H_{i j}$ is a matrix whose entries are polynomials of degree less than or equal to $d$, and such that

1. $H_{i j}(x)=H_{j i}(x)$,

2. $\sum_{k} H_{k k}(x)=0$,

3. $\sum_{k} x_{k} H_{i k}(x)=0$,

for all $1 \leq i, j \leq n$ and $x \in \mathbb{R}^{n}$. Let us denote the vector space of such matrices by $\mathcal{V}_{n}$.

If $\varepsilon_{\nu}=u_{\nu}\left(x_{\nu}\right)^{-\frac{2}{n-2}}$, the optimal pointwise estimates established in [11] allow the expansion of the interior integral of (2.1) in powers of $\varepsilon_{\nu}$, much like in the work of Aubin [2]. It turns out that the relevant correction terms are encoded in a canonical quadratic form $\mathcal{P}_{n}$ defined on $\mathcal{V}_{n}$ (see the appendix of [11] for a definition of $\mathcal{P}_{n}$ ). It follows from the arguments sketched above that the positivity of $\mathcal{P}_{n}$ is sufficient to establish the Weyl vanishing and compactness.

In the Appendix of [11] the authors analyze the eigenvalues of $\mathcal{P}_{n}$ and prove:

Proposition 2.3. The quadratic form $\mathcal{P}_{n}$, defined on $\mathcal{V}_{n}$, is positive definite if $n \leq 24$. Moreover, it has negative eigenvalues if $n \geq 25$.

The Compactness Theorem 2.1 allows us to compute the total Leray-Schauder degree of all solutions to equation (1.1), and to obtain more refined existence theorems which we now briefly discuss. 
If we choose the unit volume normalization $\int_{M} u^{\frac{2 n}{n-2}} d v_{g}=1$, the equation (1.1) becomes

$$
L_{g} u+E_{g}(u) u^{\frac{n+2}{n-2}}=0,
$$

where

$$
E_{g}(u)=-\int_{M} u L_{g} u d v_{g}=\int_{M}\left(\left|\nabla_{g} u\right|^{2}+c(n) R_{g} u^{2}\right) d v_{g} .
$$

Given $p \in\left[1, \frac{n+2}{n-2}\right]$ and $\Lambda>0$, we define a map $F_{p}: \bar{\Omega}_{\Lambda} \rightarrow C^{2, \alpha}(M)$ by

$$
F_{p}(u)=u+L_{g}^{-1}\left(E_{g}(u) u^{p}\right)
$$

where

$$
\Omega_{\Lambda}=\left\{u \in C^{2, \alpha}(M) \mid\|u\|_{C^{2, \alpha}}<\Lambda, \quad u>\Lambda^{-1}\right\}
$$

Since it follows from elliptic theory that the map $u \mapsto L_{g}^{-1}\left(E_{g}(u) u^{p}\right)$ is a compact map from $\bar{\Omega}_{\Lambda}$ into $C^{2, \alpha}(M)$, we may define the Leray-Schauder degree (see [20]) $\operatorname{deg}\left(F_{p}, \Omega_{\Lambda}, 0\right)$ of $F_{p}$ in the region $\Omega_{\Lambda}$ with respect to $0 \in C^{2, \alpha}(M)$, provided that $0 \notin F_{p}\left(\partial \Omega_{\Lambda}\right)$. The degree is an integer which counts with multiplicity the number of times that the value 0 is taken on by the map $F_{p}$. Notice that $F_{\frac{n+2}{n-2}}(u)=0$ if and only if $u$ is a solution of (2.2). The homotopy invariance of the degree tells us that

$$
\operatorname{deg}\left(F_{p}, \Omega_{\Lambda}, 0\right)=\operatorname{deg}\left(F_{1}, \Omega_{\Lambda}, 0\right),
$$

if $0 \notin F_{p}\left(\partial \Omega_{\Lambda}\right)$ for all $p \in\left[1, \frac{n+2}{n-2}\right]$. When $p=1$ the equation is linear and it is not difficult to show (see [26]) that $\operatorname{deg}\left(F_{1}, \Omega_{\Lambda}, 0\right)=-1$ for all $\Lambda$ sufficiently large.

Therefore

Theorem 2.4. Suppose that $\left(M^{n}, g\right)$ satisfies the assumptions of Theorem 2.1.

Then

$$
\operatorname{deg}\left(F_{p}, \Omega_{\Lambda}, 0\right)=-1
$$

for all $\Lambda$ sufficiently large and $p \in\left[1, \frac{n+2}{n-2}\right]$. 
In the case that all solutions of the Yamabe problem are nondegenerate, as will be the case for a generic conformal class of Riemannian metrics, our previous results assert that there will be a finite number of solutions to the variational problem. The strong Morse inequalities

$$
(-1)^{\lambda} \leq \sum_{\mu=0}^{\lambda}(-1)^{\lambda-\mu} C_{\mu}, \quad \lambda=0,1,2, \ldots,
$$

where $C_{\mu}$ denotes the number of solutions of Morse index $\mu$, hold in this case since they are well-known for the subcritical equations. We also obtain

Corollary 2.5. Suppose that $\left(M^{n}, g\right)$ satisfies the assumptions of Theorem 2.1, and assume that all critical points in $[g]$ are nondegenerate. Then there are a finite number of critical points $g_{1}, \ldots, g_{k}$ and we have

$$
1=\sum_{j=1}^{k}(-1)^{I\left(g_{j}\right)}
$$

where $I\left(g_{j}\right)$ denotes the Morse index of the variational problem with volume constraint.

\section{Noncompactness theorems}

The key to understand the noncompactness results is to look closely at the model case of the standard sphere $\left(S^{n}, g_{0}\right)$. As was pointed out in the introduction, the set of solutions of volume one coincides in this particular case with the set of metrics coming from the action of the conformal group on $g_{0}$, and therefore it is noncompact. We might then be tempted to ask the following question:

Is there a way of perturbing the conformal structure of the standard sphere so that the noncompactness persists?

It follows from Theorem 2.1 that this is impossible if $n \leq 24$. It comes as a surprise that the answer to this question is yes for all $n \geq 25$.

The main theorems of [6] and [7] put together give: 
Theorem 3.1. Suppose $n \geq 25$. Given any $\varepsilon>0$, there exists a smooth Riemannian metric $g$ on $S^{n}$ and a sequence of positive functions $v_{\nu} \in C^{\infty}\left(S^{n}\right)$ $(\nu \in \mathbb{N})$ with the following properties:

(i) $\left\|g-g_{0}\right\|_{C^{[1 / \varepsilon]}\left(S^{n}\right)}<\varepsilon$,

(ii) $g$ is not conformally flat,

(iii) $v_{\nu}$ is a solution of the Yamabe equation (1.1) for all $\nu \in \mathbb{N}$,

(iv) $Q\left(v_{\nu}^{\frac{4}{n-2}} g\right) \nearrow Q\left(S^{n}, g_{0}\right)$ as $\nu \rightarrow \infty$,

(v) $\sup _{S^{n}} v_{\nu} \rightarrow \infty$ as $\nu \rightarrow \infty$.

In [6] S. Brendle did the case $n \geq 52$, while the case $25 \leq n \leq 51$ was subsequently handled by Brendle and the author in [7].

The important point is that finding such sequences of solutions for a metric $g$ in a small neighborhood of $g_{0}$ can be reduced to solving a finite dimensional variational problem. This follows from a procedure known as the LyapunovSchmidt reduction which we now briefly describe.

Since the standard sphere minus a point is conformally equivalent to the Euclidean space through the stereographic projection, we can translate the problem to the Euclidean setting. In this setting the solutions of the Yamabe equation

$$
\Delta u+n(n-2) u^{\frac{n+2}{n-2}}=0
$$

on $\mathbb{R}^{n}$ are the functions

$$
u_{(\xi, \varepsilon)}(x)=\left(\frac{\varepsilon}{\varepsilon^{2}+|x-\xi|^{2}}\right)^{\frac{n-2}{2}},
$$

where $(\xi, \varepsilon) \in \mathbb{R}^{n} \times(0, \infty)$. The solutions of the equation (3.1) can be also seen as the critical points (at the same energy level) of the functional

$$
\mathcal{F}_{\delta}(u)=\int_{\mathbb{R}^{n}}\left(|\nabla u|^{2}-(n-2)^{2}|u|^{\frac{2 n}{n-2}}\right) d x
$$


restricted to the space

$$
\mathcal{E}=\left\{w \in L^{\frac{2 n}{n-2}}\left(\mathbb{R}^{n}\right) \cap W_{l o c}^{1,2}\left(\mathbb{R}^{n}\right): \int_{\mathbb{R}^{n}}|\nabla w|^{2} d x<\infty\right\} .
$$

We will consider Riemannian metrics $g$ which are perturbations of the Euclidean metric with compact support. We write $g(x)=\exp (h(x))$, where $h(x)$ is a trace-free symmetric two-tensor on $\mathbb{R}^{n}$ satisfying $h(x)=0$ for $|x| \geq 1$, and

$$
|h(x)|+|\partial h(x)|+\left|\partial^{2} h(x)\right| \leq \alpha
$$

for some small $\alpha>0$ and all $x \in \mathbb{R}^{n}$.

Although the linearization of the equation (3.1) has a kernel, it is possible to apply the Implicit Function Theorem if we restrict ourselves to the orthogonal subspace

$$
\mathcal{E}_{(\xi, \varepsilon)}=\left\{w \in \mathcal{E}: \int_{\mathbb{R}^{n}} \varphi_{(\xi, \varepsilon, k)} w d x=0 \quad \text { for } k=0,1, \ldots, n\right\},
$$

where

$$
\varphi_{(\xi, \varepsilon, 0)}(x)=\left(\frac{\varepsilon}{\varepsilon^{2}+|x-\xi|^{2}}\right)^{\frac{n+2}{2}} \frac{\varepsilon^{2}-|x-\xi|^{2}}{\varepsilon^{2}+|x-\xi|^{2}}
$$

and

$$
\varphi_{(\xi, \varepsilon, k)}(x)=\left(\frac{\varepsilon}{\varepsilon^{2}+|x-\xi|^{2}}\right)^{\frac{n+2}{2}} \frac{2 \varepsilon\left(x_{k}-\xi_{k}\right)}{\varepsilon^{2}+|x-\xi|^{2}}
$$

for $k=1, \ldots, n$.

As a consequence we can find an $(n+1)$-dimensional family of approximate solutions:

Proposition 3.2. Let $\alpha>0$ be sufficiently small, depending only on the dimension. Given $(\xi, \varepsilon) \in \mathbb{R}^{n} \times(0, \infty)$, there exists a function $v_{(\xi, \varepsilon)} \in \mathcal{E}$ such that $v_{(\xi, \varepsilon)}-u_{(\xi, \varepsilon)} \in \mathcal{E}_{(\xi, \varepsilon)}$ and

$$
\int_{\mathbb{R}^{n}}\left(\left\langle\nabla v_{(\xi, \varepsilon)}, \nabla \psi\right\rangle_{g}+\frac{n-2}{4(n-1)} R_{g} v_{(\xi, \varepsilon)} \psi-n(n-2)\left|v_{(\xi, \varepsilon)}\right|^{\frac{4}{n-2}} v_{(\xi, \varepsilon)} \psi\right)=0
$$

for all test functions $\psi \in \mathcal{E}_{(\xi, \varepsilon)}$. Moreover, we have the estimate

$$
\begin{aligned}
& \left\|v_{(\xi, \varepsilon)}-u_{(\xi, \varepsilon)}\right\|_{\mathcal{E}} \\
& \leq C\left\|\Delta_{g} u_{(\xi, \varepsilon)}-\frac{n-2}{4(n-1)} R_{g} u_{(\xi, \varepsilon)}+n(n-2) u_{(\xi, \varepsilon)}^{\frac{n+2}{n-2}}\right\|_{L^{\frac{2 n}{n+2}}\left(\mathbb{R}^{n}\right)},
\end{aligned}
$$

where $C$ is a constant that depends only on $n$. 
The problem reduces to finding critical points of the finite-dimensional functional $\mathcal{F}_{g}: \mathbb{R}^{n} \times(0, \infty) \rightarrow \mathbb{R}$, given by

$$
\mathcal{F}_{g}(\xi, \varepsilon)=\int_{\mathbb{R}^{n}}\left(\left|\nabla v_{(\xi, \varepsilon)}\right|_{g}^{2}+\frac{n-2}{4(n-1)} R_{g} v_{(\xi, \varepsilon)}^{2}-(n-2)^{2}\left|v_{(\xi, \varepsilon)}\right|^{\frac{2 n}{n-2}}\right) d x .
$$

The construction of the counterexample relies on a glueing procedure based on some local model metrics. The model metrics $g(x)=\exp (h(x))$ are such that

$$
h_{i k}(x)=\mu \lambda^{2 m} f\left(\lambda^{-2}|x|^{2}\right) \sum_{p, q} W_{i p k q} x_{p} x_{q}
$$

for $|x| \leq \rho$, where $\mu, \lambda, \rho$ are positive constants satisfying $\mu \leq 1$ and $\lambda \leq \rho \leq 1$, $f$ is a polynomial, and $W: \mathbb{R}^{n} \times \mathbb{R}^{n} \times \mathbb{R}^{n} \times \mathbb{R}^{n} \rightarrow \mathbb{R}$ is a nontrivial multi-linear form which satisfies all the algebraic properties of the Weyl tensor. It is also necessary that

$$
2 \operatorname{deg}(f)+2<\frac{n-2}{2} .
$$

These choices make it possible to approximate the energy function $\mathcal{F}_{g}(\xi, \varepsilon)$ at appropriate scales by an auxiliary function $F(\xi, \varepsilon), \xi \in \mathbb{R}^{n}, \varepsilon \in(0, \infty)$, and we are left with the algebraic problem of finding a polynomial $f$ such that $F(\xi, \varepsilon)$ has a strict local minimum at $(0,1)$.

Notice that

$$
\mu \lambda^{2 m} f\left(\lambda^{-2}|x|^{2}\right) \sum_{p, q} W_{i p k q} x_{p} x_{q} \in \mathcal{V}_{n}
$$

as defined in the previous section, and it turns out that the algebraic problem of finding $f$ can be solved when the quadratic form $\mathcal{P}_{n}$ has negative directions. It is proven in [6] that $f$ can be chosen of degree 1 for all $n \geq 52$, and in [7] that it can be chosen of degree 3 for all $25 \leq n \leq 51$.

The counterexamples $g(x)=\exp (h(x))$ are obtained by glueing infinite copies of the local models supported in small disjoint balls placed along the $x_{1}$ axis. The $N$-th ball has radius $1 /\left(2 N^{2}\right)$ and is centered at $y_{N}=\left(\frac{1}{N}, 0, \ldots, 0\right) \in$ $\mathbb{R}^{n}, N \in \mathbb{N}$. If $\eta: \mathbb{R} \rightarrow \mathbb{R}$ is a smooth cutoff function such that $\eta(s)=1$ for 
$s \leq 1$ and $\eta(s)=0$ for $s \geq 2$, the two-tensor $h(x)$ is given by

$$
h_{i k}(x)=\sum_{N=N_{0}}^{\infty} \eta\left(4 N^{2}\left|x-y_{N}\right|\right) 2^{-\left(m+\frac{1}{8}\right) N} f\left(2^{N}\left|x-y_{N}\right|^{2}\right) H_{i k}\left(x-y_{N}\right),
$$

where $y_{N}=\left(\frac{1}{N}, 0, \ldots, 0\right) \in \mathbb{R}^{n}, m=\operatorname{deg}(f)$, and $N_{0}$ is sufficiently large.

Since the metric $g$ is in local model form in each of the infinitely many balls $B_{1 /\left(2 N^{2}\right)}\left(y_{N}\right)$, we can apply the Lyapunov-Schmidt reduction infinitely many times to obtain a sequence $v_{\nu}$ of solutions to the Yamabe equation as in Theorem 3.1.

We should notice that even though the Weyl tensor of these counterexamples vanishes to all orders at the blow-up point $\left(0 \in \mathbb{R}^{n}\right)$, recent work of the author ([19]) shows that they can be perturbed to provide counterexamples to the Weyl Vanishing Conjecture as well.

\section{References}

[1] Ambrosetti, A.; Malchiodi, A., A multiplicity result for the Yamabe problem on $S^{n}$, J. Funct. Anal., 168, (1999), 529-561.

[2] Aubin, T., Équations différentielles non linéaires et probléme de Yamabe concernant la courbure scalaire, J. Math. Pures Appl., 55 (1976), 269-296.

[3] Berti, M.; Malchiodi., A., Non-compactness and multiplicity results for the Yamabe problem on $S^{n}$, J. Funct. Anal., 180 (2001), 210-241.

[4] Brendle, S., On the conformal scalar curvature equation and related problems, Surveys in Differential Geometry XII, (2008).

[5] Brendle, S., Convergence of the Yamabe flow in dimension 6 and higher, Invent. Math., 170, (2007), 541-576.

[6] Brendle, S., Blow-up phenomena for the Yamabe equation, J. Amer. Math. Soc., 21 (2008), 951-979, 2008. 
[7] Brendle, S.; Marques, F. C., Blow-up phenomena for the Yamabe equation II, J. Differential Geom., 81 (2009), 225-250.

[8] Druet, O., Compactness for Yamabe metrics in low dimensions, Int. Math. Res. Not., 23 (2004), 1143-1191.

[9] Druet, O.; Hebey, E., Blow-up examples for second order elliptic PDEs of critical Sobolev growth, Trans. Amer. Math. Soc., 357 (2004), 1915-1929.

[10] Hebey, E.; Vaugon, M., Le problème de Yamabe équivariant, Bull. Sci. Math., 117 (1993), 241-286.

[11] Khuri, M. A.; Marques, F. C.; Schoen, R. M., A Compactness Theorem for the Yamabe Problem, J. Differential Geom., 81 (2009), 143-196.

[12] Khuri, M. A.; Marques, F. C.; Schoen, R. M., Details of Calculations from the Appendix, http://math.stanford.edu/ schoen/yamabe-paper/, (2007).

[13] Lee, J.; Parker, T., The Yamabe problem, Bull. Amer. Math. Soc., 17 (1987), 37-91.

[14] Li, Y.; Zhang, L., Compactness of solutions to the Yamabe problem II, Calc. Var. and PDEs, 25 (2005), 185-237.

[15] Li, Y.; Zhang, L., Compactness of solutions to the Yamabe problem III, J. Funct. Anal., 245 (2) (2006), 438-474.

[16] Li, Y.; Zhu, M., Yamabe type equations on three dimensional Riemannian manifolds, Communications in Contemporary Math., 1 (1999), 1-50.

[17] Lohkamp, J., The higher dimensional positive mass theorem I, Preprint, (2006).

[18] Marques, F. C., A priori estimates for the Yamabe problem in the nonlocally conformally flat case, J. Differential Geom., 71 (2005), 315-346. 
[19] Marques, F. C., Blow-up examples for the Yamabe problem, Preprint, (2008).

[20] Nirenberg, L., Topics in Nonlinear Functional Analysis, Courant Institute publication, 1973-74.

[21] Obata, M., The conjectures on conformal transformations of Riemannian manifolds., J. Diff. Geom., 6 (1972), 247-258.

[22] Pollack, D., Nonuniqueness and high energy solutions for a conformally invariant scalar curvature equation, Comm. Anal. and Geom., 1 (1993), $347-414$.

[23] Schoen, R., Conformal deformation of a Riemannian metric to constant scalar curvature, J. Differential Geometry, 20 (1984), 479-495.

[24] Schoen, R., Courses at Stanford University, (1989).

[25] Schoen, R., Variational theory for the total scalar curvature functional for Riemannian metrics and related topics, "Topics in Calculus of Variations", Lecture Notes in Mathematics, Springer-Verlag, New York, v. 1365, (1989).

[26] Schoen, R., On the number of constant scalar curvature metrics in a conformal class, Differential Geometry: A symposium in honor of Manfredo Do Carmo (H. B. Lawson and K. Tenenblat, eds.), Wiley, (1991), 311-320.

[27] Schoen, R., A report on some recent progress on nonlinear problems in geometry, Surveys in Differential Geometry, 1 (1991), 201-241.

[28] Schoen, R.; Yau, S.-T., On the proof of the positive mass conjecture in General Relativity, Comm. Math. Phys., 65 (1979), 45-76.

[29] Schoen, R., Yau, S.-T., Conformally flat manifolds, Kleinian groups, and scalar curvature, Invent. Math., 92 (1988), 47-71. 
[30] Schoen, R.; Yau, S.-T., Lectures on Differential Geometry, Conference Proceedings and Lecture Notes in Geometry and Topology, International Press Inc., (1994).

[31] Schoen, R., Zhang, D., Prescribed scalar curvature on the n-sphere, Calc. Var. and PDEs, 4 (1996), 1-25.

[32] Trudinger, N., Remarks concerning the conformal deformation of Riemannian structures on compact manifolds, Ann. Scuola Norm. Sup. Pisa Cl. Sci., 22 (3) (1968), 165-274.

[33] Witten, E., A new proof of the positive energy theorem, Comm. Math. Phys., 80 (1981), 381-402.

[34] Yamabe, H., On a deformation of Riemannian structures on compact manifolds, Osaka Math. J., 12 (1960), 21-37.

Instituto de Matemática Pura e Aplicada (IMPA)

Estrada Dona Castorina 110

22460-320, Rio de Janeiro - RJ - Brazil

E-mail: coda@impa.br 